\title{
Measuring effectiveness of washing methods for corrosion control of archaeological iron: problems and challenges
}

\section{Watkinson*}

\begin{abstract}
The individual chloride content of 116 archaeological iron nails from Romano British and Medieval sites in Wales is reported. The meaning and value of chloride concentration recorded as weight of chloride in object/object weight is discussed in relation to reporting the effectiveness of washing methods designed to remove chloride from archaeological iron. This is theoretically compared to the concentration value weight of chloride in object/metal surface area of object and the difficulty of quantitatively determining the success of washing methods as stability enhancers is discussed. It is concluded that assessing the impact of residual chloride on post-treatment corrosion of archaeological objects has the potential to offer the most significant guide to treatment success.
\end{abstract}

Keywords: Iron, Archaeology, Corrosion, Chloride, Treatment, Washing, Assessment

\section{This paper is part of a special issue on corrosion of archaeological and heritage artefacts}

\section{Introduction}

Preserving archaeological materials as part of cultural heritage is considered important in modern day societies and no greater challenge exists than preventing the postexcavation corrosion of archaeological iron. Corrosion in terrestrial burial environments normally produces objects that have a metal core covered by a dense corrosion product layer (DPL), which is overlaid by a more voluminous and less dense transformed layer containing iron corrosion and soil (TL). ${ }^{1}$ The DPL/TL layers range from being quite thin, where they cover substantial iron cores mirroring the original size of the object, to thick layers that overlie thin needle-like iron cores. For some objects, all metallic iron is lost and only DPL remains.

The DPL is heterogeneous, normally comprising a dense $\alpha \mathrm{FeOOH}$ matrix containing $\mathrm{Fe}_{2} \mathrm{O}_{3}$ and $\mathrm{Fe}_{3} \mathrm{O}_{4}$ strips and it contains microcracks. ${ }^{1}$ During burial chlorides are drawn in from soils as counter ions for $\mathrm{Fe}^{2+}$ generated at anode sites on the metal surface beneath the DPL layer ${ }^{2}$ and they predominantly exist in solution. ${ }^{1,2}$ Small pockets of akaganeite $(\beta \mathrm{FeOOH})$ have been detected in the DPL and larger amounts at the metal/DPL interface, along with ferrous hydroxychloride $\left[\beta \mathrm{Fe}_{2}(\mathrm{OH})_{3} \mathrm{Cl}\right]$, which is thought to be a precursor for $\beta \mathrm{FeOOH}$ formation. ${ }^{3,4} \beta \mathrm{FeOOH}$ forms in the presence of chloride, which it adsorbs onto its surface and occludes in its crystal structure. ${ }^{5-7}$

School of History and Archaeology, Cardiff University, Cardiff, South Glamorgan CF10 3EU, UK

*Corresponding author, email watkinson@ cardiff.ac.uk
Post-excavation atmospheric corrosion of archaeological iron involves chloride acting as an electrolyte and the generation of $\mathrm{FeOOH}$ polymorphs including $\beta \mathrm{FeOOH} .{ }^{8-10}$ This detaches DPL corrosion layers, disfiguring the object. Dry storage of excavated archaeological iron will concentrate chloride at the metal surface and either $\mathrm{FeCl}_{2} \cdot 4 \mathrm{H}_{2} \mathrm{O}$ or $\mathrm{FeCl}_{2} \cdot 2 \mathrm{H}_{2} \mathrm{O}$ may form. A change to damp storage conditions can hydrolyse these compounds to $\beta \mathrm{FeOOH}$. Both $\mathrm{FeCl}_{2} \cdot 4 \mathrm{H}_{2} \mathrm{O}$ and $\beta \mathrm{FeOOH}$ can corrode iron at low relative humidity. ${ }^{9,11,12}$

Controlling the post-excavation corrosion caused by soluble chloride and chloride bearing corrosion products is essential to prevent break-up of archaeological iron objects. Controlling corrosion normally involves either chloride passivation by humidity control or removal of chlorides followed by controlled storage. Aqueous washing techniques are often employed in attempts to solvate and remove chloride. Chloride removed during treatment is normally quantified, ${ }^{13-18}$ but it is unclear what information this supplies regarding the success of a treatment or the post-treatment stability of objects. Historically, once no further chloride was being extracted into the wash solution, it was assumed the iron was free of all chloride, but post-treatment digestion of expendable objects reveals they often have significant chloride residues. ${ }^{15-17}$ Studies attempting to determine 'percentage chloride extraction efficiency' for a given treatment with fixed variables such as treatment time, temperature, solution chemistry and concentration, have revealed erratic and unpredictable chloride extraction for many treatment methods. ${ }^{14-18}$ Efficiency has been defined as the percentage of the total chloride within an object which was removed by treatment, where total chloride content is determined by object digestion. ${ }^{14-16}$ Assessing 


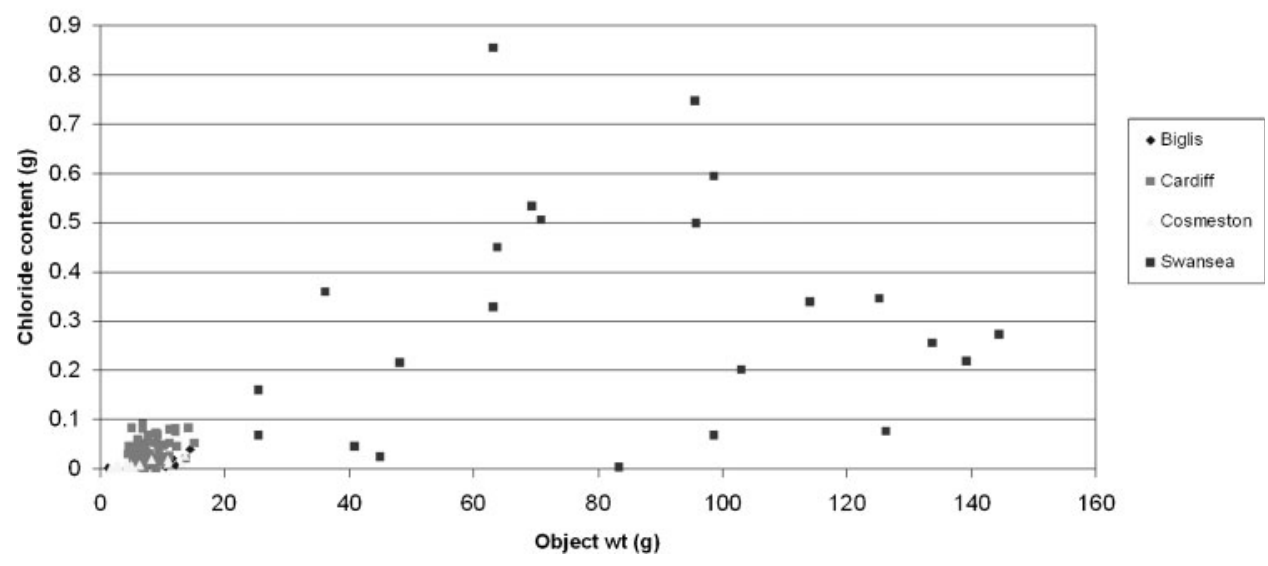

1 Chloride content of 116 archaeological iron objects from four sites

treatment success rarely focused on the quantity of chloride remaining in objects and its potential significance for object stability.

This paper discusses some of the challenges associated with quantifying treatment efficiency for washing methods and the impact of treatments on the corrosion rate of objects by:

(i) reporting the chloride content of 116 archaeological iron objects from terrestrial sites

(ii) examining the relationship between the chloride content of terrestrial archaeological iron, its weight and its surface area

(iii) discussing chloride chemistry in objects and its relevance to chloride extraction

(iv) considering how measuring chloride extracted by treatment contributes to assessing treatment success.

\section{Experimental method}

The chloride content of 116 archaeological iron objects that had undergone washing treatments ${ }^{16,17,19}$ was determined. They all retained metal cores as determined by Xradiography and were from Welsh archaeological sites. They had been stored in uncontrolled storage environments for several years. Cardiff (70 objects), Newport (23 objects) and Cosmeston (15 objects) are medieval sites and Biglis (8 objects) is Romano British. Cardiff and Swansea objects had been treated individually by a range of aqueous chloride extraction methods (Table 1) reported elsewhere. ${ }^{16,17}$ Biglis and Cosmeston had been treated in various sequences by alkaline sulphite, boiling deionised water, Soxhlet (aq.) and pressurised steam. ${ }^{19}$ Posttreatment, each object was placed in $5 \mathrm{M} \mathrm{HNO}_{3}$ in a watch glass covered beaker and heated on a water bath daily (not overnight) for up to 3 weeks. Smaller objects digested completely and all corrosion products and some iron core dissolved on the larger objects.

Chloride measurement for neutral aqueous systems was by an EIL 7065 specific ion meter $( \pm 1 \mathrm{mV})$ using $10 \mathrm{~mL}$ samples buffered $1: 10$ with $0 \cdot 5 \mathrm{M} \mathrm{HN}_{4} \mathrm{CH}_{3} \mathrm{COO} /$ $\mathrm{CH}_{3} \mathrm{COOH}$. The meter was calibrated with five standard solutions at half day intervals. Modification of this method for different treatments involved preparing the sample according to the treatment procedure used:

(i) Digestion - Cardiff and Swansea: neutralise drop-wise with $0 \cdot 5 \mathrm{M} \mathrm{NaOH}$ and wash precipitate free of any chloride into neutralised solution

(ii) $\mathrm{NaOH} / \mathrm{Na}_{2} \mathrm{SO}_{3}$ : neutralise drop-wise $5 \mathrm{M}$ $\mathrm{H}_{2} \mathrm{SO}_{4}$, heat daily on water bath in a covered beaker for 3 days

(iii) $\mathrm{NaOH}$ : neutralise drop-wise $5 \mathrm{M} \mathrm{H}_{2} \mathrm{SO}_{4}$

(iv) $\mathrm{NaSO}_{3}$ : heat daily on water bath in a covered beaker for 3 days

(v) Digestion - Biglis and Cosmeston: $2 \mathrm{M} \mathrm{HNO}_{3}$ $\mathrm{Fe}^{2+}$ removal in ion exchange column, neutralisation with $2 \mathrm{M} \mathrm{NaOH}$.

All methods were tested using known amounts of chloride to establish their reproducibility and the precipitate formed in (i) was digested post-washing and shown to be chloride free. ${ }^{17}$ Calculation determined the amount of; chloride removed by treatment; post-treatment residual chloride; total chloride content of each object.

\section{Results}

The weight and total chloride content of the 116 archeological iron objects is shown in Fig. 1, with details of objects weighing less than $16 \mathrm{~g}$ in Fig. 2.

Table 1 Treatment procedures for archaeological iron*

\begin{tabular}{lc}
\hline Treatment & Time, days \\
\hline $0.5 \mathrm{M} \mathrm{NaOH} / \mathrm{Na}_{2} \mathrm{SO}_{3}$ : RT sealed PE container & 60 \\
$0.5 \mathrm{M} \mathrm{NaOH}$ deoxygenated: RT in open container in larger box purged with nitrogen & 60 \\
Aqueous Soxhlet wash deoxygenated: purged with nitrogen 75-90 C & $60-48$ cycles per day \\
Aqueous wash deoxygenated: RT in open container in larger box purged with nitrogen & 60 \\
$0.5 \mathrm{M} \mathrm{Na} \mathrm{SO}_{3}:$ RT & 60 \\
$0.5 \mathrm{M} \mathrm{NaOH}:$ RT & 60 \\
Aqueous wash: RT static & 60 \\
Soxhlet wash open to atmosphere & 60
\end{tabular}

*RT: room temperature. 


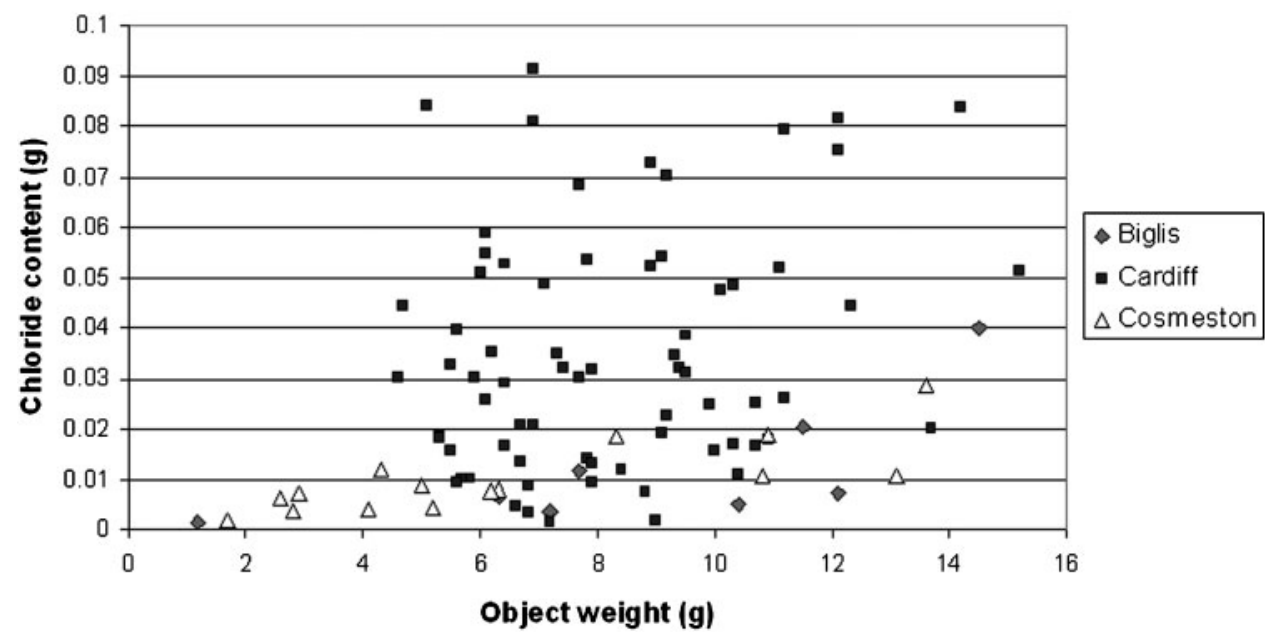

2 Chloride content of 92 archaeological iron objects from Cardiff, Biglis and Cosmeston weight $<16 \mathrm{~g}$

The weight of chloride in each object appears to be unpredictable and generally unrelated to object weight (Figs. 1 and 2). All objects $>16 \mathrm{~g}$ are from the Swansea site, while objects $<16 \mathrm{~g}$ are from Cardiff, Biglis and Cosmeston. For Biglis and Cosmeston it appears that increasing object weight produces increased chloride content. However, there are too few objects to statistically investigate this apparent trend and neither Swansea nor Cardiff sites produce the same trend for much bigger object populations (Fig. 2). Also, objects of a similar weight from the same site can have greatly differing chloride content (Swansea and Cardiff sites, Figs. 1 and 2 ). The ceiling content of chloride in objects from Cosmeston and Biglis is lower than that for Cardiff, yet their objects lie within the same weight range as the Cardiff objects (Figs. 1 and 2). As expected the largest amount of chloride is contained in the heaviest objects, yet heavy objects can also contain small amounts of chloride similar to many lighter objects.

Detailed examination shows a $95.6 \mathrm{~g}$ iron object contains $0.747 \mathrm{~g}$ of chloride, but a $98.6 \mathrm{~g}$ object contains $10 \times$ less chloride $(0.068 \mathrm{~g})$. Similarly, a $6.9 \mathrm{~g}$ object contains $0.091 \mathrm{~g}$ of chloride and a $6.8 \mathrm{~g}$ object contains $11 \times$ less chloride $(0.008 \mathrm{~g})$. The maximum chloride content is a remarkable $268 \times$ the minimum for objects $>16 \mathrm{~g}$ and $60 \times$ for samples $<16 \mathrm{~g}$ (Figs. 1 and 2 ). All objects $<16 \mathrm{~g}$ in weight contain less than $0 \cdot 1 \mathrm{~g}$ of chloride and, while objects $>16 \mathrm{~g}$ contained up to $0.85 \mathrm{~g}$ of chloride, 7 of the 23 objects $>16 \mathrm{~g}$ fall within the same chloride weight range as samples below $16 \mathrm{~g}$.

\section{Discussion}

\section{Chloride content of iron objects}

Figures 1 and 2 examine chloride content as a weight value, but chloride associated with archaeological iron is mostly reported as the concentration value; weight of chloride in object/weight of object. Plotting this chloride concentration ( $\mathrm{ppm}$ ) for each of the 116 objects against their weight shows how concentration varies with weight of object (Fig. 3). Using a chloride concentration value apparently allows comparison between objects, as lighter objects containing less chloride than heavier objects can have similar chloride concentrations. The chloride concentrations within objects appear to cover a similar range irrespective of object weight; some lighter objects have the highest chloride concentrations, while some heavier objects have the lowest. Comparing sites, Biglis and Cosmeston objects contain lower concentrations of chloride, while objects from the Cardiff and Swansea sites contain similar chloride concentrations that extend over a very wide range of values.

It might be thought that a measure of object stability would be to compare chloride concentrations within objects, using the premise that there will be more free chloride to act as an electrolyte in objects containing high chloride concentrations, irrespective of their weight. This comparison ignores the surface area of metal available for corrosion and thus the concentration of chloride at the metal surface. Calculating chloride concentration per unit surface area of metallic iron is likely to offer better insight into corrosion rate and object stability because theory, measurement and observation suggest that most chloride is held at the metal surface. $^{4,9,10}$ This is often localised as chloride nests ${ }^{4}$ and their activation in the atmosphere causes objects to break up. For iron objects of the same weight in similar environments, flat thin objects will offer a larger surface area for corrosion to draw in chloride ions, as compared to compact rectangular or cylindrical shaped objects. Consequently, flat objects might be expected to contain more chloride in relation to their weight, mineralise more quickly and potentially disappear entirely in burial contexts when compared to the more compact object morphologies. Unfortunately, calculating the metal surface area on archaeological iron is unrealistic, as it is hidden beneath the DPL and is not flat surface. Only computed tomography with texture analysis can venture to examine this surface without destroying the object by removing all its information retaining corrosion layers. However, experimental studies that link object stability, metal surface area and post-treatment chloride content, could offer useful insight into treatment effectiveness.

A theoretical study of the influence of surface area can be considered. All objects from the sites examined here are either complete nails or nail fragments with roughly generic shapes, being forged from iron bloom to form wrought iron of density $\sim 7.86 \mathrm{~g} \mathrm{~cm}^{-3}$ at $20^{\circ} \mathrm{C}$. This 
Relative concentration of chloride per object

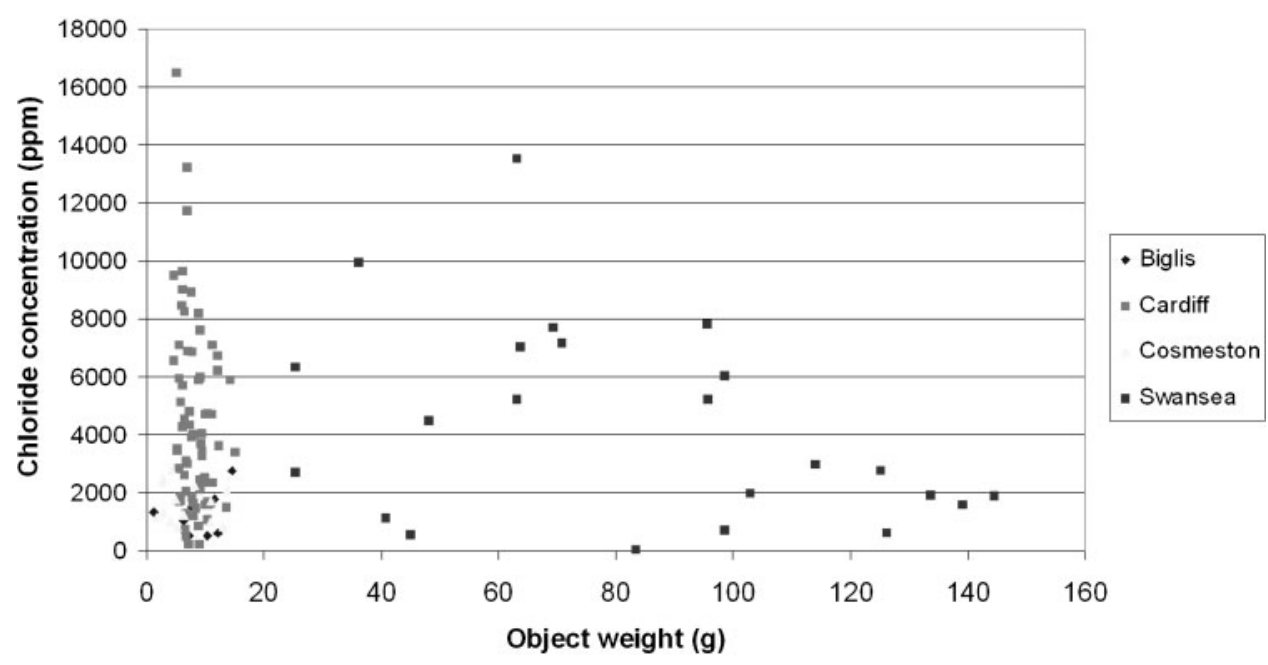

3 Chloride in object (ppm) recorded as weight of chloride in object/weight of object plotted against weight of object

commonality of shape can be used to consider how surface areas vary as a function of weight and how this may influence concentration measurements. For $10 \mathrm{~cm}$ long wrought iron bars of varied square cross-section (Table 2), lighter bars offer a larger surface area on which corrosion can occur relative to their weight (Fig. 4). Potentially, with all other factors being equal, they can theoretically pull in more chloride counter ions as a function of their weight. This means that lighter objects could show high concentrations of chloride using weight chloride in object/weight object as a concentration determinant. Figure 3 reveals that some of the lightest objects have high chloride concentrations.

Contrary to the expectation of conservators who are responsible for treatment and preservation of archaeological iron, the concentration value weight of chloride in object/weight object may not allow comparisons between object stability on the pretext that similar concentrations of chloride indicate similar object stability. Furthermore, no studies have quantitatively linked ppm chloride to object stability in any comparative manner. Theoretically comparing objects of similar generic shape with the same ppm chloride concentration but with differing weights, offers insight into what information weight of chloride in object/weight object can provide about object stability. In Table 3 objects of similar morphological shape cited in Table 2 are considered to contain 1000 ppm chloride, calculated as weight chloride in object/weight object and their total chloride content is calculated and then related to their surface area.

For this given generic shape, heavier objects have much higher concentrations of chloride per unit surface

Table 2 Surface area, volume and weight of $10 \mathrm{~cm}$ bars of wrought iron of differing square cross-sections

\begin{tabular}{llll}
\hline $\begin{array}{l}\text { Cross-section: } \\
\text { side of square, } \mathbf{c m}\end{array}$ & $\begin{array}{l}\text { Volume, } \\
\mathbf{c m}^{\mathbf{3}}\end{array}$ & $\begin{array}{l}\text { Surface } \\
\text { area, } \mathbf{c m}^{\mathbf{2}}\end{array}$ & $\begin{array}{l}\text { Weight, } \\
\mathbf{g}\end{array}$ \\
\hline 0.25 & 0.625 & $10 \cdot 130$ & $\begin{array}{l}4.910 \\
0.50\end{array}$ \\
0.50 & 2.500 & 21 & 19.65 \\
0.75 & $5 \cdot 625$ & $31 \cdot 250$ & $44 \cdot 125$ \\
1.00 & 10 & 42 & 78.6 \\
1.5 & $25 \cdot 50$ & 65.06 & $200 \cdot 6$ \\
2.0 & 40 & 88 & 314.4 \\
\hline
\end{tabular}

area than lighter objects, yet their chloride concentration measured as weight of chloride in object/weight of object is the same. Since the heavier objects contain higher concentrations of chloride per unit area of metal surface they might be expected corrode more rapidly and lose shape faster than those containing lower concentrations.

Comparing chloride concentrations in objects as a function of object weight may not provide a relative comparison of stability, but it does offer a crude scale of stability for each object; low chloride concentrations are likely to mean increased object stability. This theoretical comparison between surface areas is undeveloped, as the localisation of chloride in pits and other factors need to be considered to produce a more advanced model. Also, even if small objects corrode more slowly, their localised loss of DPL may soon destroy their shape, whereas large objects may be able to accept some local DPL loss and retain their archaeological value for longer periods. Neither does the model consider the form of chloride present. Also, archaeological objects beginning life with similar generic shapes may be reduced to thin metal cores overlaid by thick DPL layers, which may contribute either significantly or negligibly to object weight according to the object size. Clearly, using the ratio weight of chloride/weight of object as a comparator of object stability and treatment success is limited.

\section{Chloride form}

Corrosion of archaeological iron is not only influenced by chloride quantity and object morphology, but also by chloride form within the object, as soluble chloride acts as an electrolyte. $\beta \mathrm{FeOOH}$ can corrode iron via its readily soluble surface adsorbed chloride ${ }^{2,11,12}$ and some of the chloride from within its crystal structure can be solvated to be available for corrosion processes. ${ }^{6}$ While not all the chloride from within $\beta \mathrm{FeOOH}$ can be removed by washing, ${ }^{6,7}$ it appears that washing either dramatically reduces or stops its ability to corrode iron. ${ }^{11,12}$ Therefore, objects containing similar amounts of chloride, but in differing forms can be expected to corrode at differing rates. No quantified comparisons exist between the corrosivity of soluble chloride and similar amounts of chloride associated with $\beta \mathrm{FeOOH}$, but an object containing most of its chloride as $\beta \mathrm{FeOOH}$ might reasonably be 


\section{Weight of object versus surface area}

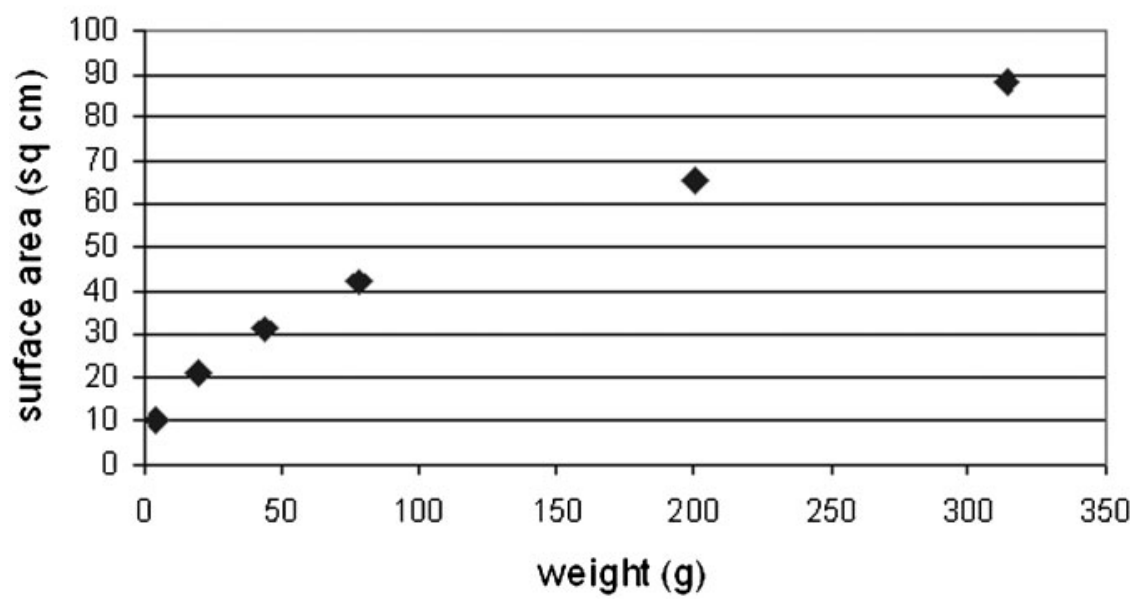

\section{Object weight versus object surface area for $10 \mathrm{~cm}$ long wrought iron bars with various square cross sections}

expected to corrode less quickly than an object containing its chloride entirely in soluble forms, as not all the chloride in $\beta \mathrm{FeOOH}$ is available for corrosion processes.

Paradoxically, although it seems retention of chloride as $\beta \mathrm{FeOOH}$ reflects greater object stability, when compared to objects with chloride in soluble forms, this is not the case when considering the survival of the object as an archaeological record. The driver for controlling the quantity of $\beta \mathrm{FeOOH}$ present on archaeological iron will be the amount of post-excavation corrosion. The quantities of chloride and $\mathrm{Fe}^{2+}$ ions present will influence $\beta \mathrm{FeOOH}$ formation. While sufficiently high concentrations of chloride are required to form $\beta \mathrm{FeOOH}$, low concentrations of $\mathrm{Fe}^{2+}$ favour the formation of $\alpha \mathrm{FeOOH}$ and $\gamma \mathrm{FeOOH}$ even in the presence of high chloride levels. ${ }^{20}$ Removing some of the soluble chloride from the corrosion process by 'locking it' into the structure of newly formed $\beta \mathrm{FeOOH}$ requires iron corrosion to generate $\mathrm{Fe}^{2+}$ to form the $\beta \mathrm{FeOOH}$. This iron dissolution weakens the integrity of bonding at the $\mathrm{DPL} / \mathrm{Fe}$ interface and the resulting $\beta \mathrm{FeOOH}$ growth creates physical pressure on the DPL, destroying the physical integrity of objects which nullifies their heritage value. In light of this destruction, discussing form and relative amount of chloride compounds present on objects may appear irrelevant, but it is relevant to determining the effectiveness of washing methods for increasing the stability of iron.

\section{Assessing treatment success}

Chloride quantity and treatment effectiveness

When treating archaeological iron objects using washing methods the chloride content of individual objects is unknown both before and after treatment. Clearly objects are not normally dissolved post-treatment to determine their chloride content and there is no knowledge of how much chloride is removed from a single object since cost, time and object numbers normally dictate that iron small finds are treated in batches, although large objects are treated individually. Knowing collectively the amount of chloride removed from 300 objects does not inform whether any single object contained large or small amounts of chloride. Consider all 116 objects in Fig. 1 treated as one single batch; residual chloride will certainly differ dramatically between objects. Additionally, the chloride forms and their relative quantities are unknown before and after treatment, yet chloride form and quantity will influence post-treatment corrosion rate.

Assessments have shown that treatments produce wide ranges of extraction efficiencies, with deoxygenated alkaline systems generally acknowledged as being the most effective (Table 4). ${ }^{15,16}$

What information can the standard conservation practice of measuring chloride removed from objects provide for persons treating objects? First, it can indicate treatment end points by detecting when no further chloride is being extracted. Second, the quantity of chloride removed can offer a guide as to whether there are large or small amounts of soluble chloride in an object or an object batch. It cannot provide information as to how much chloride objects will retain. Applying best and worst treatment efficiencies reported in Table 4 to the range of objects in Fig. 1 reveals that post-treatment chloride concentration in objects

Table 3 Calculated concentration of chloride per unit surface area for iron objects containing $1000 \mathrm{ppm}$ chloride determined by ratio weight of chloride in object/weight of object

\begin{tabular}{|c|c|c|c|c|}
\hline $\begin{array}{l}\text { Cross-section: } \\
\text { side of square, } \mathrm{cm}\end{array}$ & $\begin{array}{l}\text { Surface } \\
\text { area, } \mathrm{cm}^{2}\end{array}$ & $\begin{array}{l}\text { Weight } \\
\text { object, g }\end{array}$ & $\begin{array}{l}\text { Weight chloride } \\
\text { in object with concentration } \\
\text { of } 1000 \mathrm{ppm} \mathrm{Cl}^{-}, \mathrm{g}\end{array}$ & $\begin{array}{l}\text { Concentration of } \\
\text { chloride per unit surface } \\
\left.\text { area, (wt- } \% \mathrm{Cl}^{-} / \mathrm{SA}\right), \mu \mathrm{g}\end{array}$ \\
\hline 0.25 & $10 \cdot 130$ & 4.910 & 0.004910 & 484 \\
\hline 0.50 & $21 \cdot 0$ & $19 \cdot 65$ & 0.019650 & 936 \\
\hline 0.75 & $31 \cdot 250$ & $44 \cdot 125$ & 0.044125 & 1412 \\
\hline $1 \cdot 00$ & $42 \cdot 0$ & 78.60 & 0.078600 & 1871 \\
\hline 1.5 & 65.06 & $200 \cdot 60$ & $0 \cdot 200600$ & 3083 \\
\hline $2 \cdot 0$ & $88 \cdot 0$ & $314 \cdot 40$ & 0.314400 & 3573 \\
\hline
\end{tabular}


Table 4 Reported treatment efficiencies represented as percentage of total chloride in object extracted by treatment ${ }^{15,16}$

\begin{tabular}{llclc}
\hline & \multicolumn{2}{c}{ Efficiency of chloride extraction as percentage of total chloride in object, \% } \\
\cline { 2 - 5 } Treatment & Average & Std. dev. & Worst & $\begin{array}{c}\text { Number } \\
\text { objects } \\
\text { in sample }\end{array}$ \\
\hline $0.5 \mathrm{M} \mathrm{Na} \mathrm{SO}_{3} / \mathrm{NaOH}$ & 87 & 8 & 68 & 95 \\
$0.5 \mathrm{M} \mathrm{NaOH}-$ aerated & 64 & 16 & 32 & 87 \\
Soxhlet - water & 81 & 12 & 67 & 99 \\
deaerated nitrogen gas & & & 62 & 17 \\
Water - deaerated static & 74 & 11 & 12 & 91 \\
Water - aerated static & 34 & 15 & 10 & 58 \\
\hline
\end{tabular}

can potentially vary significantly. Considering these limitations, it would appear that measuring the effectiveness of treatments is best served, not by applying experimentally determined chloride extraction efficiencies for a given treatment nor by measuring the chloride extracted, but from experimental study of the corrosion capacity of the residual chloride. Variation in residual chloride levels within objects and their individual physical uniqueness and importance as an archaeological record, means that any such assessment should examine the corrosion response of individual objects relative to their post-treatment chloride content. This shifts assessment of treatment success away from the amount of chloride that is removed during treatment, to the amount of chloride remaining in the object and its influence on post-treatment corrosion. To assess this, it is important to consider the form of the chloride before and after treatment.

\section{Chloride form and treatment effectiveness}

The relative quantities of chloride counter ions, $\beta \mathrm{FeOOH}, \beta \mathrm{Fe}_{2}(\mathrm{OH})_{3} \mathrm{Cl}$ and $\mathrm{FeCl}_{2} 4 \mathrm{H}_{2} \mathrm{O}$ will influence how much chloride washing methods remove from archaeological iron. It is unlikely that all soluble chloride counter ions will diffuse out during aqueous washing, due to object morphology and chloride location at the metal surface beneath dense DPL layers. These pockets of soluble chloride will likely offer the greatest post-treatment corrosion threat. Washing $\beta \mathrm{FeOOH}$ in water and alkali will remove its surface adsorbed chloride ${ }^{6,7,17}$ which removes its hygroscopicity and prevents it corroding iron. ${ }^{6,21}$ Removal of chloride occluded in tunnels within $\beta \mathrm{FeOOH}$ may not be expected to be complete during washing, but it has been shown that simple aqueous washing for $72 \mathrm{~h}$ can remove much of the tunnel located chloride from the $\beta \mathrm{FeOOH}$ without transforming it to other corrosion products ${ }^{6}$ and that most chloride lies within the $\beta \mathrm{FeOOH}$ tunnel structure for chloride content below 6 mass- $\%$. Similarly, hot $\left(50^{\circ} \mathrm{C}\right)$ and cold deionised water washes lasting $60 \mathrm{~h}$ were shown to rapidly remove chloride (41 and 28\% respectively) from $\beta \mathrm{FeOOH}(4.5$ mass- $\%$ chloride $),{ }^{17}$ although this offers a less complete washing process. Excess $\mathrm{OH}^{-}$in solution is reported to remove chloride from tunnels within $\beta \mathrm{FeOOH},{ }^{22}$ which gives alkaline washing systems and advantage over other methods. However, there are many synthesis routes for $\beta \mathrm{FeOOH}$, which can have several forms that hold differing amounts of chloride. All these factors are likely to influence washing effectiveness.

Although washed $\beta \mathrm{FeOOH}$ should offer no corrosion threat, $^{12,22}$ the reported metastability of $\beta \mathrm{FeOOH}^{13}$ could release its internal chloride sometime in the future. Even though this metastability is in doubt, ${ }^{12}$ it would be preferable if washing treatments transformed $\beta \mathrm{FeOOH}$ and released its chloride. While transformation has received limited attention in conservation literature, heated alkaline conditions characteristic of washing treatments have the potential to transform $\beta \mathrm{FeOOH} .{ }^{5,18,23,24}$ $\beta \mathrm{FeOOH}$ transformation in $\mathrm{KOH}$ at $70^{\circ} \mathrm{C}$ was influenced by $\mathrm{OH}^{-}$concentration producing either $\alpha \mathrm{FeOOH}$ or $\alpha \mathrm{FeOOH}$ and $\mathrm{Fe}_{2} \mathrm{O}_{3}{ }^{24} \mathrm{~A}$ sample of $\beta \mathrm{FeOOH}(4.5$ mass $-\%$ chloride) boiled in deionised water and left to stand for 4 months was found to have transformed to $18 \%$ $\beta \mathrm{FeOOH} ; 14 \% \quad \alpha \mathrm{FeOOH} ; 70 \% \quad \mathrm{Fe}_{3} \mathrm{O}_{4} \cdot{ }^{17}$ Pressurised $\mathrm{NaOH}$ treatment produced full transformation of a $\beta \mathrm{FeOOH} / \alpha \mathrm{FeOOH} / \mathrm{Fe}_{3} \mathrm{O}_{4} / \gamma \mathrm{FeOOH}$ mix to $\alpha \mathrm{FeOOH}$, $\mathrm{Fe}_{2} \mathrm{O}_{3}$ and $\mathrm{Fe}_{3} \mathrm{O}_{4}{ }^{23}$ Boiling with water for six days was found to convert $\beta \mathrm{FeOOH}$ to $\alpha \mathrm{Fe}_{2} \mathrm{O}_{3}{ }^{25}$ and at room temperature $\beta \mathrm{FeOOH}$ in water slowly transformed to $\alpha \mathrm{FeOOH}{ }^{6}$ Sodium hydroxide reduced the chloride content of $\beta \mathrm{FeOOH}$ containing 4.5 mass- $\%$ chloride to 0.656 mass- $\%$ suggesting some transformation had occurred. ${ }^{17} \beta \mathrm{FeOOH}$ transformation patterns are varied, unpredictable and merit further study to understand the potential of treatments for removing chloride. It is clear that chloride extraction efficiencies (Table 4) must also record corrosion product transformations and these will influence the quantity and form of residual chloride in objects.

\section{How stable are treated objects?}

Discussion here considered the use and meaning of the ratio weight of chloride in object/weight of object for the experimental study of treatment efficiency and, by extrapolation, treatment effectiveness. Given the erratic nature of chloride extraction (Table 4) and the large differences in chloride content of objects, it is suggested that determining the effectiveness of treatments lies with the response of treated objects to their environments, rather than focusing on the amount of chloride removed from objects. Quantities of chloride remaining in objects will vary greatly making it essential to focus on how these residues influence iron corrosion. By how much do washing methods improve object stability and how does this relate to optimum storage conditions? There are pertinent resource related questions to answer, such as whether storage humidity can be relaxed from the very low percentages required to stop corrosion in the presence of chloride bearing corrosion products, ${ }^{11,21}$ to higher values once objects have been washed. Do washed objects continue to corrode over the same humidity range as unwashed objects, but at slower rates, or is their relative humidity corrosion threshold raised? A more difficult question is how to define object lifespan? The quantity and influence of residual chloride on corrosion, the transformation of $\beta \mathrm{FeOOH}$, corrosion rates of chloride infested iron and object longevity are currently being investigated at Cardiff University as 
part of an AHRC funded PhD and an AHRC/EPSRC Science and Heritage large grants award.

An obvious and final question is whether it is worth washing objects, given the treatment unknowns and the resource issues involved? A simple answer to support washing is that lowering chloride levels will reduce corrosion rate. However, this has to be considered in relation to cost benefit, which cannot be assessed without further research. There remain other factors to consider, such as the effect of treatment chemical residues including $\mathrm{Na}_{2} \mathrm{SO}_{3}, \mathrm{NaOH}$ and products of their reaction with $\mathrm{Fe}^{2+}$ and the atmosphere. ${ }^{26}$

\section{Conclusion}

There are wide variations between the chloride content of archaeological objects, even when they are from the same archaeological site. Measuring the chloride removed from archaeological objects in ppm as a function of object weight does not offer a tool for comparing object stability or measuring treatment success, due to morphological, weight and chemical composition differences between objects. New experimental methodologies need to be adopted to examine treatment success. These should focus on the quantity and form of chloride remaining in objects, as well as the corrosion rate of treated objects in various relative humidities. Overall, the vast range of variables involved in treating archaeological iron and the chemical and morphological uniqueness of each object makes quantitative assessment of treatment success a challenging proposition.

\section{References}

1. D. Neff, E. Vega, P. Dillmann and M. Descostes: in 'Corrosion of metallic artefacts; investigation, conservation and prediction for long-term behavior', (ed. P. Dillmann et al.), European Federation of Corrosion Publications Number 48, 41-76; 2007, Cambridge, CRC Press, Woodhead Publishing.

2. S. Turgoose: in 'Conservation of iron', (ed. R. Clarke and S. Blackshaw), Monograph No. 53, 1-8; 1982, London, National Maritime Museum.

3. F. Zucci, G. Morigi and V. Bertolasi: 'Beta iron oxide hydroxide formation in localised active corrosion of iron artifacts', in 'Corrosion and metal artifacts: a dialogue between conservators, and archaeologists and corrosion scientists', (ed. F. B. Brown), National Bureau of Standards Special Publication 479, 103-105; 1977, NBS Washington DC.
4. S. Reguer, P. Dillmann, F. Mirambet and J. Susini: in 'Corrosion of metallic artefacts; investigation, conservation and prediction for long-term behavior', (ed. P. Dillmann et al.), European Federation of Corrosion Publications Number 48, 170-189; 2007, Woodhead Publishing Ltd, CRC Press.

5. R. M. Cornell and U. Schwertmann: 'The iron oxides: structure, properties, reactions occurrences and uses', 2nd edn; 2003, Weinheim, Wiley-VCH.

6. S. Reguer, F. Mirambet, E. Dooryhee, J.-L. Hodeau, P. Dillmann and P. Lagarde: Corros. Sci., 2009, 51, 2795-2802.

7. K. Stahl, K. Nielsen, J. Jiang, B. Lebech, J. C. Hanson, P. Norby and J. van Lanschot: Corros. Sci., 2003, 45, 2563-2575.

8. S. Reguer, D. Neff, L. Bellot-Gurlet and P. Dillmann: J. Raman Spectros., 2007, 38, 389-397.

9. S. Turgoose: Stud. Conserv., 1982, 27, 97-101.

10. L. S. Selwyn, P. J. Sirois and V. Argyropoulous: Stud. Conserv., 1999, 44, 217-232.

11. D. E. Watkinson and M. R. T. Lewis: Proc. Int. Conf. on 'Metals conservation', (ed. J. Ashton and D. Hallam), 88-103; 2004, Canberra, National Museum of Australia.

12. D. E. Watkinson and M. R. T. Lewis: in 'Materials issues in art and archaeology VII', (ed. P. B. van Diver et al.), 103-114; 2004, Warrendale, PA, Materials Research Society.

13. D. A. Scott and N. I. Seeley: 1987, 32, 73-76.

14. D. Watkinson: Stud. Conserv., 1983, 29, 85-90.

15. D. E. Watkinson: in 'Archaeological conservation and its consequences', (ed. A. Roy and P. Smith), International Institute for Conservation, London. 208-212; 1996.

16. D. Watkinson and A. Al Zahrani: Conservator, 2008, 31, 75-86.

17. M. J. Drews, P. de Vivies, N. G. Gonzalez and P. Mardikian: Proc. Int. Conf. on 'Metals conservation', (ed. J. Ashton and D. Hallam), 247-260; 2004, Canberra, National Museum of Australia.

18. A. Al Zahrani: 'Chloride ion removal from archaeological iron and $\beta \mathrm{FeOOH}, \mathrm{PhD}$ thesis, Cardiff University, Cardiff, UK, 1999.

19. D. E. Watkinson: 'A comparison of chloride extraction methods for archeological ironwork', Master's dissertation, Cardiff University, Cardiff, UK, 1983.

20. I. Wiesner, B. Schmutzler and G. Eggert: in 'Metal 07: Interim Meeting of the ICOM-CC Metal Working Group', (ed. C. Degrigny et al.), Vol. 5, 'Protection of metal artefacts', 110 114; 2007, Amsterdam, Rijksmuseum.

21. D. Watkinson and M. R. T. Lewis: Stud. Conserv., 2005, 50, 241252 .

22. J. Cai, J. Liu, Z. Gao, A. Navrotsky and S. L. Suib: Chem. Mater., 2001, 13, (12), 4595-4602.

23. P. de Vivies, D. Cook, M. J. Drews, N. G. Gonzalez, P. Mardikian and J. B. Memet: in 'Metal 07: Interim Meeting of the ICOM-CC Metal Working Group', (ed. C. Degrigny et al.), Vol. 5, 'Protection of metal artefacts', 26-30; 2007, Amsterdam, Rijksmuseum.

24. R. M. Cornell and R. Giovanoli: Clays Clay Miner., 1991, 39, (2), $144-150$.

25. J. D. Bernal, D. R. Dasgupta and A. L. Mackay: Clay Mineral. Bull., 1959, 4, 15-30.

26. M. B. Rimmer and D. Watkinson: Forthcoming in ICOM-CC Metals 2010, Charleston, WV, USA, October 2010. 